Check for updates

Cite this: Chem. Sci., 2018, 9, 209

\title{
Brominated graphene as a versatile precursor for multifunctional grafting $\dagger$
}

\begin{abstract}
Heather Au, (D) Noelia Rubio (D) and Milo S. P. Shaffer (D)*
A non-destructive and versatile chemical reduction method was used to dissolve and subsequently brominate few-layer graphene sheets (FLGs); the direct covalent attachment of bromine to the graphene framework was demonstrated by X-ray photoelectron spectroscopy (XPS). The brominated few-layer graphenes (FLG-Br) provide a convenient, stable, liquid-phase precursor, suitable for the synthesis of a variety of directly functionalised graphenes. As an example, the $\mathrm{FLG}-\mathrm{Br}$ species was used to initiate atom transfer radical polymerisation (ATRP), to obtain poly(methyl methacrylate) (PMMA)-grafted graphene (FLG-PMMA), which was six times more dispersible in acetone than controls. In addition, the $\mathrm{FLG-Br}$ is active for nucleophilic substitution reactions, as illustrated by the preparation of methoxypolyethylene glycol (mPEG)- and $\mathrm{OH}$ substituted derivatives. The products were characterised by thermogravimetric analysis coupled with mass spectrometry (TGA-MS), XPS and Raman spectroscopy. Grafting ratios (GR) for these polymer-grafted materials varied between 6 and 25\%; even at these GRs, all graphene derivatives showed increased solubility in organic solvents.
\end{abstract}

Received 8th August 2017

Accepted 29th September 2017

DOI: $10.1039 / \mathrm{c} 7 \mathrm{sc} 03455 \mathrm{e}$

rsc.li/chemical-science including exposure to bromine vapour, ${ }^{26}$ long sonication ${ }^{27}$ or

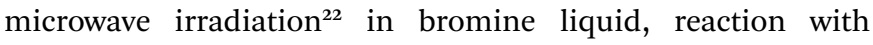
dibromocarbene, ${ }^{28}$ and photocatalytic addition using $\mathrm{N}$-bromosuccinimide as a bromine precursor. ${ }^{29}$ However, direct covalent attachment of bromine to graphene is rare and usually achieved by harsh methods such as plasma ${ }^{30}$ or microwave $\mathrm{e}^{22}$ treatment, or refluxing in bromine ${ }^{31}$ and/or on poorly exfoliated materials. Nevertheless, bromine-activated carbon species can be used in subsequent nucleophilic substitution reactions, as reported for brominated highly-oriented pyrolytic graphite (HOPG) and multi-walled carbon nanotubes (MWCNTs); for example, following plasma-chemical bromination, the $\mathrm{C}-\mathrm{Br}$ bonds underwent hydrolysis, or further grafting with 1,6-diaminohexane and (3-aminopropyl)triethoxysilane. ${ }^{30}$ Bromination of single-walled carbon nanotubes (SWCNTs) by reductive functionalisation also showed that nucleophilic substitution of sidewall bromine addends was possible, introducing 2,2,2-trifluoroethanolate and hydroxyl groups at the brominated sites. ${ }^{32}$

In general, reductive functionalisation is a versatile route to obtaining CNMs grafted with a wide variety of moieties. ${ }^{33-35}$ For graphene, in particular, charging the carbon framework can be achieved by vapour transport, ${ }^{36,37}$ direct metal (alloy) contact, ${ }^{38}$ or through the use of charge transfer agents ${ }^{39}$ to form alkalimetal graphite intercalation compound (GIC) precursors. Under appropriate conditions, in dry aprotic solvents, individual anionic 'graphenide' sheets can be dissolved and processed. Reaction with a wide variety of different electrophiles yields individualised, covalently-grafted graphene derivatives. The connectivity of the carbon lattice is retained, thereby reducing the detrimental effects that covalent grafting and
Departments of Chemistry \& Materials, Imperial College London, London, SW7 2AZ, UK.E-mail: m.shaffer@imperial.ac.uk

$\dagger$ Electronic supplementary information (ESI) available: Additional TEM, TGA-MS Raman and XPS characterisation, grafting calculations and solubility data Further supporting data may be requested from the corresponding author, but may be subject to confidentiality obligations. See DOI: 10.1039/c7sc03455e 
exfoliation can have on graphene's intrinsic mechanical and electrical properties. This method has resulted in successful grafting of alkyl, ${ }^{\mathbf{4 4 3 9 , 4 0}}$ aryl, ${ }^{\mathbf{4 1}}$ and polyethylene glycol (PEG) ${ }^{\mathbf{4 2}}$ addends, as well as hydrogenation; ${ }^{43}$ however, bromination of graphene is not yet reported by this route.

Polymer grafting can greatly improve the solubility of CNMs, additionally limiting their restacking and agglomeration on drying. ${ }^{44}$ Using polymers with defined molecular weight and an activated end group, in a 'grafting-to' approach, allows control of polymer properties; ${ }^{45}$ however, the large steric bulk of the chains tends to limit grafting density. Conversely, surface-initiated anionic polymerisation can achieve densely-grown polymers, ${ }^{46}$ but requires rigorously dry reaction conditions to allow full control over molecular weight and dispersity. Furthermore, the choice of monomers is limited to only those which are stable under reducing conditions. Alternative grafting-from approaches such as atom transfer radical polymerisation (ATRP) afford more control over polymer growth, ${ }^{47}$ whilst retaining a high graft density. Previous examples of ATRP from nanomaterial surfaces include the polymerisation of styrene, methyl methacrylate (MMA) and glycidyl methacrylate from graphene oxide (GO), CNTs and boron nitride nanotubes (BNNTs). ${ }^{8,48-52}$ In these procedures, bromine-containing initiator molecules are grafted first (usually via multi-step reactions), rather than directly attaching an active bromine atom to the surface.

The aim of this work was to prepare directly brominated graphene by reductive chemistry as an air-stable precursor for a variety of further transformations. As an illustration, brominated few-layer graphene (FLG-Br) was used to obtain poly(methyl methacrylate) (PMMA)-grafted graphene (FLG-PMMA), via ATRP; in this approach, the grafting reaction is initiated directly from the graphene framework, without an intervening molecular linker. In addition, the potential for nucleophilic substitution was explored using OH-substituted reagents poly(ethylene glycol) methyl ether (mPEG) and water. In comparison with direct reactions with graphenide, both methods offer greater versatility, reflected in their accessibility to a wide range of potential functionalities, including those unstable to reducing conditions. This approach provides an easy means to access modified fewlayer graphene (FLG) with improved and tunable dispersibility, critical in bulk applications requiring compatibility with common organic solvents and polymer matrices.

\section{Experimental}

\section{Materials}

FLG was obtained from Cambridge Nanosystems and used without any further purification. Tetrahydrofuran (THF), dried in-house in a solvent-drying tower packed with alumina, was degassed via a freeze-pump-thaw method then further dried over 20 vol\% 4 A activated molecular sieves. Naphthalene (99\%, SigmaAldrich) was dried under vacuum in the presence of phosphorus pentoxide before use. Sodium (99.95\%, ingot) and bromine (reagent grade) were purchased from Sigma-Aldrich and used as received. MMA (99\%, Sigma-Aldrich) was passed through an alumina column to remove stabilisers, before degassing via freeze-pump-thaw and drying over 20 vol\% $4 \quad \begin{aligned} & \AA \\ & \text { activated }\end{aligned}$ molecular sieves. Acetone ( $\geq 99.9 \%$, Sigma-Aldrich) was distilled over $\mathrm{CaH}_{2}$ and stored under nitrogen. Immediately before use, both monomer and solvent were purged with nitrogen for $30 \mathrm{~min}$. Copper(I) bromide (CuBr, 98\%, Sigma-Aldrich) was purified by washing with glacial acetic acid, followed by 2-propanol, and then dried under vacuum. ${ }^{47} N, N, N^{\prime}, N^{\prime \prime}, N^{\prime \prime}$-Pentamethyldiethylenetriamine (PMDETA, 99\%) and copper(II) bromide $\left(\mathrm{CuBr}_{2}, 99 \%\right)$ were obtained from Sigma-Aldrich and used as received. mPEG $\left(M_{\mathrm{W}}\right.$ 2000 Da, Sigma Aldrich) was dried under vacuum with phosphorus pentoxide prior to use. Dichloromethane (VWR) was used as received.

\section{Procedures}

Preparation of sodium-naphthalide solution. A stock sodium-naphthalide solution was prepared to allow for accurate, simple addition of sodium to dried FLG. $23 \mathrm{mg}$ ( $1 \mathrm{mmol})$ sodium and $128 \mathrm{mg}$ ( $1 \mathrm{mmol})$ dried naphthalene were added to $10 \mathrm{~mL}$ degassed anhydrous THF in a $\mathrm{N}_{2}$-filled glove box, and stirred for 1 day until all sodium had dissolved, forming a darkgreen solution.

Synthesis of FLG-Br. A Young's tube containing FLG $(60 \mathrm{mg}$, $5 \mathrm{mmol}$ carbon) and a glass stirrer bar was heated at $400{ }^{\circ} \mathrm{C}$ for $1 \mathrm{~h}$ under vacuum, and placed in the glove box. $4.16 \mathrm{~mL}$ of the sodium-naphthalide solution was added to the Young's tube and the concentration of FLG in THF adjusted to $0.1 \mathrm{M}$ by addition of $45.8 \mathrm{~mL}$ degassed anhydrous $\mathrm{THF}(\mathrm{C} / \mathrm{Na}=12,[\mathrm{Na}]=0.008 \mathrm{M})$. The suspension was stirred for 1 day, then ultrasonicated for $5 \mathrm{~min}$, before connecting to an Ar-filled Schlenk line and purging 3 times. After cooling to $0{ }^{\circ} \mathrm{C}, 1 \mathrm{~mL}$ (19.4 mmol) liquid bromine was added dropwise slowly, under positive pressure of argon. The reaction was allowed to reach room temperature and stirred for 1 day. After bubbling dry $\mathrm{O}_{2} / \mathrm{N}_{2}(20 / 80 \%)$ through the solution for $15 \mathrm{~min}$, the solution was stirred overnight under dry $\mathrm{O}_{2} / \mathrm{N}_{2}$ to quench any remaining charges on the functionalised graphene. The mixture was filtered through a $0.1 \mu \mathrm{m}$ PTFE membrane (Millipore), and washed thoroughly with $N, N$-dimethylacetamide (DMAc) and THF to remove any residual sodium, naphthalene and bromine by-products. The product FLG-Br was obtained after drying overnight under vacuum.

Quenched control. A control experiment excluding the addition of bromine was conducted in the same way as before. The quenched product was washed and dried thoroughly, prior to characterisation.

Bromine adsorption control (FLG $\left.+\mathbf{B r}_{2}\right)$. A Young's tube containing FLG (15 mg, $1.25 \mathrm{mmol}$ carbon) and a glass stirrer bar was heated at $400{ }^{\circ} \mathrm{C}$ for $1 \mathrm{~h}$ under vacuum, and placed in the glove box. $12.5 \mathrm{~mL}$ degassed anhydrous THF was added and the suspension was stirred for 1 day. Following ultrasonication for $5 \mathrm{~min}$, the flask was connected to an Ar-filled Schlenk line and purged three times. After cooling to $0{ }^{\circ} \mathrm{C}, 0.25 \mathrm{~mL}(4.85 \mathrm{mmol})$ liquid bromine was added dropwise under positive pressure of argon. The reaction was allowed to reach room temperature and stirred for 1 day. After bubbling dry $\mathrm{O}_{2} / \mathrm{N}_{2}$ through the solution for $15 \mathrm{~min}$, the solution was stirred overnight under dry $\mathrm{O}_{2} / \mathrm{N}_{2}$. The mixture was filtered through a $0.1 \mu \mathrm{m}$ PTFE membrane, and 
washed thoroughly with DMAc and THF. The product was obtained after drying overnight under vacuum.

Synthesis of FLG-PMMA via ATRP. A Schlenk flask containing FLG-Br (20 mg, $0.02 \mathrm{mmol} \mathrm{Br}$ ) and a stirrer bar was heated at $80{ }^{\circ} \mathrm{C}$ under vacuum for $2 \mathrm{~h} .20 \mathrm{~mL}$ dry acetone was added, and the mixture ultrasonicated for $10 \mathrm{~min}$ to disperse the FLG-Br. The suspension was degassed by three freeze-pump-thaw cycles. Separately, $\mathrm{CuBr}(8 \mathrm{mg}, 0.056 \mathrm{mmol})$ and $\mathrm{CuBr}_{2}(1.26 \mathrm{mg}$, $0.0056 \mathrm{mmol}$ ) were added to a Schlenk flask equipped with a stirrer bar, which was previously evacuated and flushed with nitrogen. The flask was degassed and filled with nitrogen three times and then left under nitrogen. Subsequently, MMA (1 mL, $9.36 \mathrm{mmol})$ and PMDETA $(0.012 \mathrm{~mL}, 0.057 \mathrm{mmol})$ were added and the solution was stirred until the $\mathrm{Cu}$ complex had formed. The mixture was degassed by three freeze-pump-thaw cycles, then introduced to the FLG-Br solution using a dry, air-free syringe, and the flask placed in an oil bath and stirred at $50{ }^{\circ} \mathrm{C}$ for $2 \mathrm{~h}$. The flask was then removed from the oil bath and the reaction stopped by exposing to air and dilution with THF. The mixture was filtered through a $0.1 \mu \mathrm{m}$ PTFE membrane and washed with copious amounts of acetone and THF, then dried under vacuum.

ATRP control (FLG + MMA control). In a similar reaction and washing sequence to before, as-received FLG $(20 \mathrm{mg})$, rather than FLG-Br, was used as the initiator in a polymerisation control reaction.

Synthesis of FLG-PEG via nucleophilic substitution. A roundbottomed flask containing FLG-Br $(20 \mathrm{mg}, 0.02 \mathrm{mmol} \mathrm{Br}$ ) and a stirrer bar was heated at $80{ }^{\circ} \mathrm{C}$ under vacuum for $2 \mathrm{~h} .15 \mathrm{~mL}$ dry $\mathrm{THF}$ and $5 \mathrm{~mL}$ dichloromethane were added and the mixture ultrasonicated for 10 min to disperse the FLG-Br. mPEG (100 $\mathrm{mg}, 0.05 \mathrm{mmol}$ ) was added then the reaction mixture was stirred at $40{ }^{\circ} \mathrm{C}$ for $2.5 \mathrm{~h}$. The suspension was filtered through a $0.1 \mu \mathrm{m}$ PTFE membrane and washed with copious amounts of THF, water and ethanol. The product FLG-PEG was obtained after drying under vacuum.

Synthesis of FLG-OH via nucleophilic substitution. FLG-OH was obtained by an identical procedure using water $(3 \mathrm{~mL})$ as the hydroxyl source, instead of mPEG. The product was washed and dried as before, prior to characterisation.

\section{Equipment and characterisation}

Thermogravimetric analysis coupled with mass spectrometry (TGA-MS) was performed using a Mettler Toledo TGA/DSC 1 instrument integrated with a Hiden HPR-20 QIC EGA mass spectrometer under nitrogen atmosphere. Samples were held at $100{ }^{\circ} \mathrm{C}$ for $30 \mathrm{~min}$, then heated from $100{ }^{\circ} \mathrm{C}$ to $850{ }^{\circ} \mathrm{C}$ at

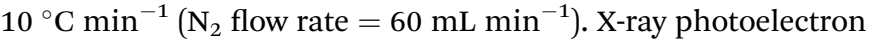
spectroscopy (XPS) data were recorded using a $\mathrm{K}$-alpha $^{+}$XPS spectrometer equipped with an MXR3 $\mathrm{Al} \mathrm{K \alpha}$ monochromated Xray source $(h \nu=1486.6 \mathrm{eV})$. X-ray gun power was set to $72 \mathrm{~W}(6$ $\mathrm{mA}$ and $12 \mathrm{kV}$ ). Charge compensation was achieved with the FG03 flood gun using a combination of low energy electrons and the ion flood source. Survey scans were acquired using $200 \mathrm{eV}$ pass energy, $1 \mathrm{eV}$ step size and $100 \mathrm{~ms}$ (50 ms $\times 2$ scans) dwell times. All high resolution spectra were acquired using
$20 \mathrm{eV}$ pass energy, $0.1 \mathrm{eV}$ step size and $1 \mathrm{~s}(50 \mathrm{~ms} \times 20$ scans $)$ dwell times. Samples were prepared by pressing the sample onto carbon-based double-sided tape. Pressure during measurement acquisition was $\leq 1 \times 10^{-8}$ mbar. Atomic compositions were calculated from averaged spectra obtained from at least 3 areas per sample. Raman spectra were collected on a Renishaw inVia micro-Raman (1000-3000 $\left.\mathrm{cm}^{-1}\right)$, using a $50 \mathrm{~mW} 532 \mathrm{~nm}$ laser at $10 \%$ laser power. Statistical Raman data were obtained from measurements carried out in Streamline mode of at least 500 areas per sample. Samples were prepared by drop casting dispersions on a glass slide or silicon wafer. UV-Vis-NIR absorption spectra were measured with a Perkin Elmer Lambda 950 UV/Vis spectrophotometer, using a quartz cuvette with $1 \mathrm{~cm}$ pathlength. Ultrasonication was performed using an ultrasonic cleaner (USC300T, $80 \mathrm{~W}$ ). Transmission electron microscopy (TEM) was carried out using a JEOL 2100Plus TEM at $200 \mathrm{kV}$ operating voltage. Samples were prepared on 300 copper mesh holey carbon grids (Agar Scientific) by drop-casting dilute graphene dispersions onto a grid supported by filter paper and drying under vacuum.

\section{Results and discussion}

\section{Synthesis of brominated graphene}

Following a previously established functionalisation sequence, ${ }^{39}$ reduced FLG was prepared by treatment with sodium naphthalide in THF, with a charging ratio (number of framework carbon atoms per sodium atom, C/Na) of 12 , and an absolute sodium concentration of $0.01 \mathrm{M}$. This reductive treatment generates a black, graphenide-containing dispersion, via electrostatically-driven exfoliation. The resulting charged graphene sheets were reacted with bromine liquid to yield brominated graphene (Scheme 1). Stable bromine-GICs are wellknown in the literature, where bromine molecules adsorb within the interlayer galleries; ${ }^{25,53-55}$ reductive activation of FLG to form a reactive intermediate is therefore required for covalent functionalisation to take place. ${ }^{32}$ The FLG starting material is already reasonably exfoliated ( $<5$ layers), with a crumpled morphology, and small lateral flake size of $0.25-1 \mu \mathrm{m}$ (Fig. S1 $\dagger$ ). The flake surfaces and edges are accessible for functionalisation, whilst non-covalently adsorbed bromine is less likely to remain trapped between graphene layers after processing; compared to larger, flatter sheets, quantification of grafting is more straightforward.

TGA-MS under nitrogen indicates successful covalent functionalisation of the FLG starting material (Fig. 1a). The weight loss in the region between 200 and $450{ }^{\circ} \mathrm{C}$ can be assigned to the detachment of bromine addends ${ }^{29,32}$ from mass fragments $\mathrm{m} / \mathrm{z}$ 79,81 , which show a characteristic isotopic distribution of $1: 1$. Bromine is known to intercalate graphite to form stable $\mathrm{Br}_{2}$ GICs, but desorption occurs at relatively low temperatures (80$\left.180{ }^{\circ} \mathrm{C}\right) .{ }^{56}$ A reference experiment mixing bromine with uncharged FLG (FLG $+\mathrm{Br}_{2}$ ) showed that bromine was fully removed by the same washing procedure as used for the functionalised product, suggesting that bromine species detected are covalently-bound (Fig. S2a $\dagger$ ). In FLG-Br, MS fragments attributed to residual THF were detected, due to some solvent 


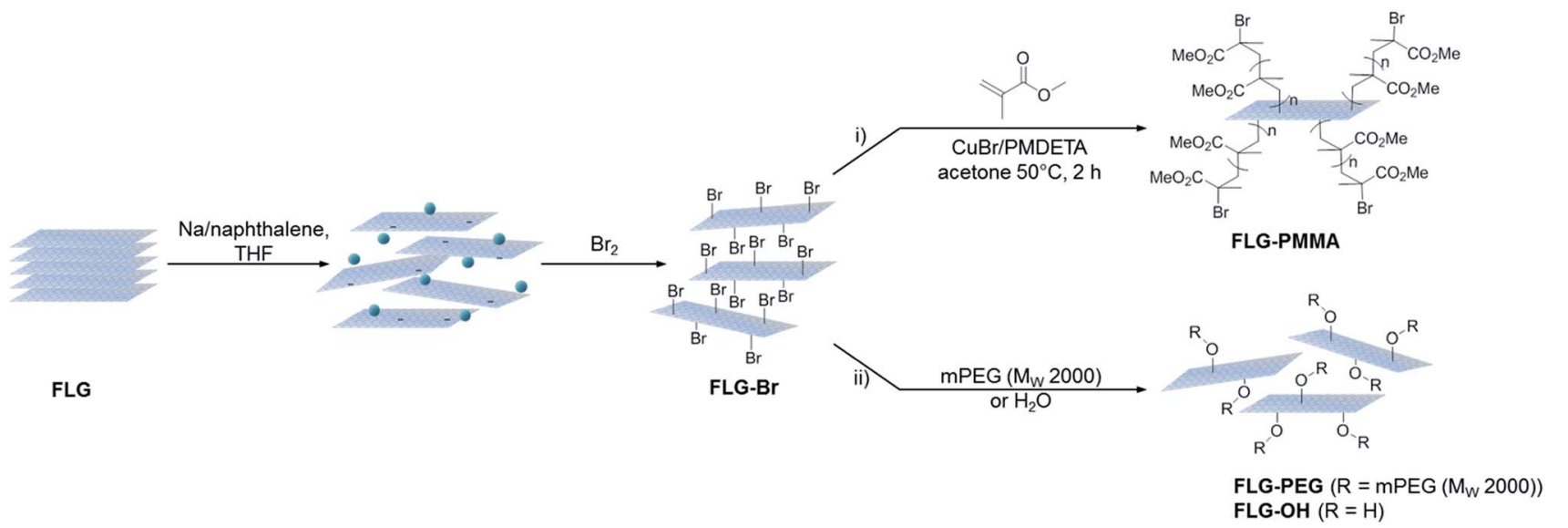

Scheme 1 Bromine functionalisation of FLG to FLG-Br via reduction with sodium naphthalide in THF, followed by (i) synthesis of FLG-PMMA via ATRP; (ii) nucleophilic substitution of FLG-Br with mPEG or water, yielding FLG-PEG and FLG-OH, respectively.

trapped within the sample (Fig. S2b†); it is therefore difficult to quantify the degree of grafting definitively from TGA alone. However, from the mass loss during pyrolysis (8.4 wt\%), an upper bound for the grafting ratio is $9.3 \mathrm{wt} \%$, corresponding to a C/Br of 71 (Table 1 ).

Further evidence for covalent functionalisation was obtained by XPS. In the wide survey XPS spectrum of FLG-Br (Fig. 1b), the major peak at $284.6 \mathrm{eV}$ arises from $\mathrm{C} 1 \mathrm{~s}$ of $\mathrm{sp}^{2}$ hybridised carbon. Additional peaks corresponding to $\mathrm{Br} 3 \mathrm{~s}, 3 \mathrm{p}_{1 / 2} / 3 \mathrm{p}_{3 / 2}$ and $3 \mathrm{~d}$ appear at $257.1,190.1 / 184.1$ and $70.2 \mathrm{eV}$, respectively. In the high resolution bromine spectrum (Fig. 1e), the deconvoluted
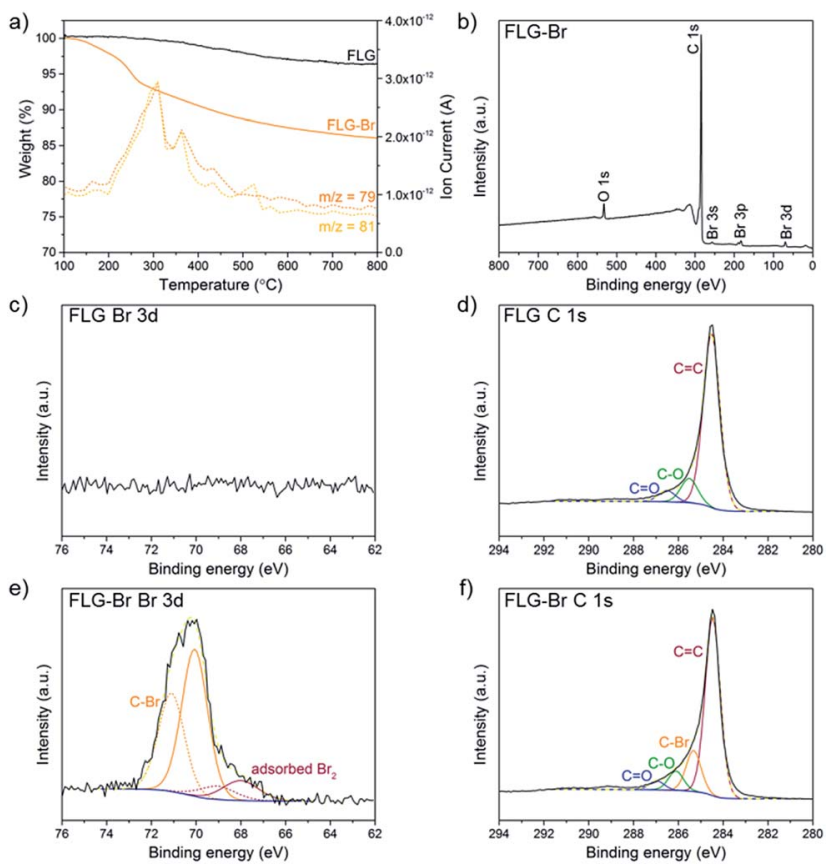

Fig. 1 (a) TGA-MS profile of FLG (black) and FLG-Br (orange), $m / z 79$ $81-\mathrm{Br}^{+}$; (b) wide survey XPS spectrum of FLG-Br; (c) core level $\mathrm{Br} 3 \mathrm{~d}$ spectrum for FLG; (d) core level C 1s spectrum for FLG; (e) core level $\mathrm{Br}$ $3 d$ spectrum for $F L G-B r$, dashed lines denote $3 d_{3 / 2}$, solid $3 d_{5 / 2}$; (f) core level $\mathrm{C}$ 1s spectrum for FLG-Br.
$\mathrm{Br} 3 \mathrm{~d}$ peaks at 70.1 and $71.1 \mathrm{eV}$ are characteristic of covalentlybound $\mathrm{Br}^{26}$ although a small fraction $(\sim 12 \%)$ of adsorbed bromine or residual sodium bromide was detected, around $68.5 \mathrm{eV},{ }^{22,57-59}$ consistent with these species being intercalated between graphene layers. A corresponding $\mathrm{C}-\mathrm{Br}$ peak at $285.3 \mathrm{eV}$ can be identified in the deconvoluted $\mathrm{C}$ 1s core-level spectrum of FLG-Br, ${ }^{28}$ but is absent in FLG, confirming that $\mathrm{Br}$ atoms have been covalently attached to the graphene sheets (Fig. 1d and f). Additional peaks at 286.1 and $286.9 \mathrm{eV}$ are assigned to $\mathrm{C}-\mathrm{O}$ and $\mathrm{C}=\mathrm{O}$, respectively, also present in the starting material. No Br $3 \mathrm{~d}$ peak was detected in the as-received sample (Fig. 1c) or control (Fig. S3†). The XPS data therefore present strong evidence for covalent $\mathrm{Br}$ attachment, consistent with reports in the literature concerning bromine functionalisation of graphite by microwave irradiation ${ }^{15}$ and of various CNMs by plasma-chemical treatment. ${ }^{22,28,30}$ Analysis of the higher resolution data indicates a composition of 0.9 at $\%$ covalent $\mathrm{Br}$ to 95.6 at\% $\mathrm{C}$ (and 3.4 at\% O) after functionalisation, giving a C/Br of 108 and grafting ratio of $6.2 \mathrm{wt} \%$ (Table 1). The relative amount of oxygen and carbon does not vary substantially compared to FLG starting material ( 96.5 at\% C, 3.5 at\% O) suggesting that the THF content is insignificant. The GRs by TGA and XPS are in good agreement, given the differences in surface sensitivity, and are comparable to the degree of grafting achieved for alkylated and arylated graphenes obtained

Table 1 XPS atomic compositions and grafting ratios of functionalised FLGs

\begin{tabular}{llllll}
\hline Sample & at\% C & at\% O & at\% Br & GR (TGA) & GR (XPS) \\
\hline FLG & 96.5 & 3.5 & - & - & - \\
FLG-Br & 95.6 & 3.4 & 0.9 & 9.3 & 6.2 \\
FLG-PMMA & 92.6 & 6.9 & 0.5 & 19.5 & 21.4 \\
FLG-PEG & 90.9 & 9.0 & 0.1 & 23.8 & 26.2 \\
FLG-OH & 94.7 & 4.9 & 0.4 & 6.5 & 3.8
\end{tabular}

${ }^{a}$ Grafting moiety/FLG mass ratios; in wt $\%$. For details of these calculations, see ESI. 
following similar reductive treatment. ${ }^{\mathbf{1 4 , 3 9 , 4 1}}$ The number density (number of grafted sites per unit area) of bromine calculated from XPS data $\left(3.5 \times 10^{13} \mathrm{~cm}^{-2}\right)$, therefore, also agrees well with that obtained from TGA $\left(5.4 \times 10^{13} \mathrm{~cm}^{-2}\right)$ (see ESI for calculations $\dagger$ ). This degree of functionalisation remains below the critical threshold for preserving the band structure of graphene, ${ }^{60}$ in contrast to harsher methods of bromination, which achieve stoichiometries of up to $40 \% \mathrm{Br} / \mathrm{C}$ but degrade the connectivity of the graphitic network, reflected in the significant broadening and weakening of characteristic Raman signals, and greatly decreased thermal stability. ${ }^{31}$

Raman spectroscopy is commonly used to provide semiquantitative analysis of the degree of grafting in carbon nanomaterials. In heterogeneous bulk samples, Raman mapping is necessary to obtain statistically relevant conclusions regarding the degree and distribution of functionalisation. Graphitic materials typically exhibit three characteristic bands: D band at $\sim 1350 \mathrm{~cm}^{-1}$, which is defect activated; G band at $\sim 1580 \mathrm{~cm}^{-1}$, related to the in-plane graphitic bonding; and $2 \mathrm{D}$ band at $\sim 2700 \mathrm{~cm}^{-1}$, from which information about stacking and layers can be determined. ${ }^{61}$ In general, the relative intensities of the defect and graphitic bands $\left(I_{\mathrm{D}} / I_{\mathrm{G}}\right)$ are used to estimate the number of grafted sites. Statistical Raman analysis of the bulk sample shows an $I_{\mathrm{D}} / I_{\mathrm{G}}$ ratio of $0.40 \pm 0.03$ in the FLG starting material
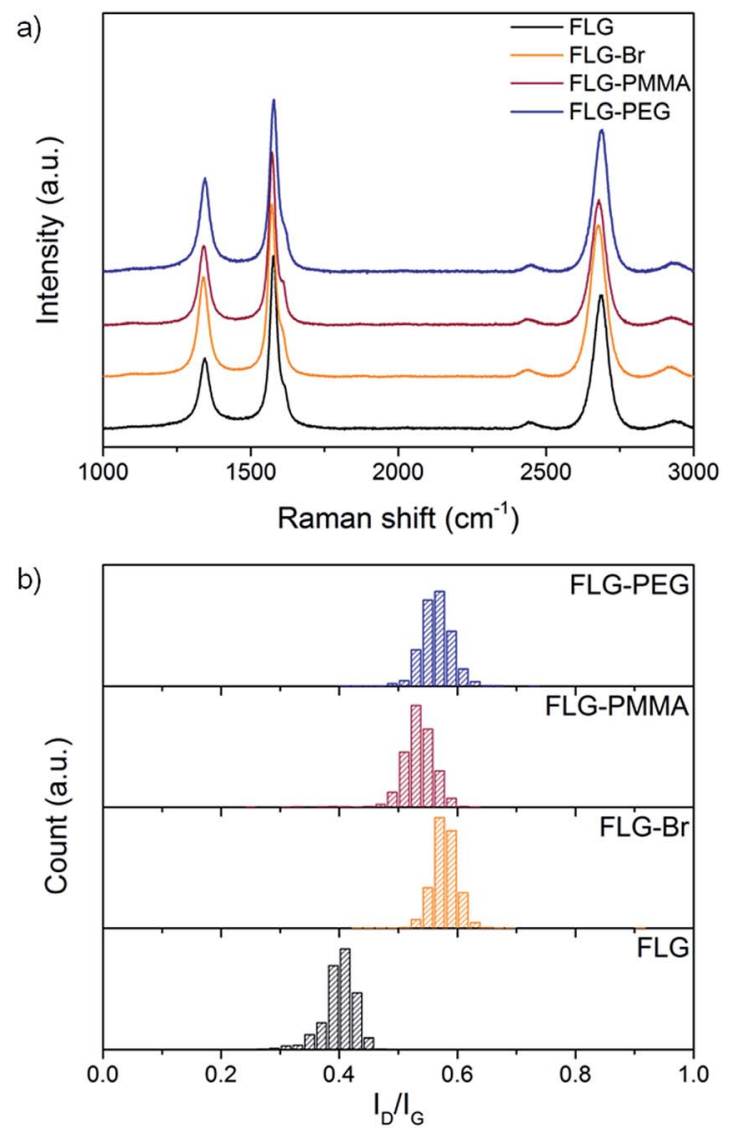

Fig. 2 (a) Averaged Raman spectra, and (b) Raman histograms of $I_{D} / I_{G}$ ratio, of FLG (black), FLG-Br (orange), FLG-PMMA (red) and FLG-PEG (blue); $\lambda_{\text {exc }}=532 \mathrm{~nm}$, spectra normalised to the $G$ peak intensity and offset for clarity.
(Fig. 2b); the large D peak arises from edge scattering, ${ }^{62}$ because many flakes are smaller than the laser spot size. For charged, unfunctionalised FLG directly quenched with dry oxygen/ nitrogen, and for FLG $+\mathrm{Br}_{2}$ control, the $I_{\mathrm{D}} / I_{\mathrm{G}}$ ratios $(0.43 \pm 0.05$ and $0.45 \pm 0.09$, respectively) remain essentially unchanged, confirming that no $\mathrm{sp}^{3}$ defects have been introduced (Fig. S4 $\dagger$ ). In contrast, $I_{\mathrm{D}} / I_{\mathrm{G}}$ increases to $0.58 \pm 0.02$ in FLG-Br (Fig. 2b orange) suggesting that bulk covalent functionalisation has indeed occurred. ${ }^{39,63}$ However, at the high grafting densities implied by the TGA and XPS data, ${ }^{\mathbf{6 4 6 5}}$ the $I_{\mathrm{D}} / I_{\mathrm{G}}$ trend might be expected to reverse, and the peaks broaden, since the defect spacing should be below the Tuinstra-Koenig limit, ${ }^{6,67}$ if uniformly distributed. Since, in fact, the spectra for FLG-Br show very well-defined peaks with narrow full width at half maximum (FWHM, $\Gamma$, Fig. 2a and Table $\mathrm{S} 1 \dagger)$, it is likely that grafting occurs in clusters, possibly nucleating from existing defects or edges, as has been proposed previously for Birch-type reductions of SWCNTs. ${ }^{68}$ Additional analysis of the $\mathrm{D}^{\prime}$ band at $\sim 1615 \mathrm{~cm}^{-1}$ shows an increase in $I_{\mathrm{D}} / I_{\mathrm{D}^{\prime}}$ after functionalisation (Fig. S5 $\dagger$ ) which is consistent with an increase in the ratio of $\mathrm{sp}^{3}$ defects to edge-type defects, ${ }^{69}$ and therefore indicates that covalent grafting occurs, with retention of lattice connectivity. The width of the $I_{\mathrm{D}} / I_{\mathrm{G}}$ ratio distribution remains essentially unchanged (Fig. 2b), suggesting that all the flakes in the sample react equally. The $2 \mathrm{D}$ peaks indicate the extent of exfoliation and are discussed below.

\section{FLG-Br as a precursor to polymer-grafted graphene}

Polymer grafting can improve the dispersibility of graphene in common organic solvents or matrices, ${ }^{70}$ and potentially enhance stress-transfer in nanocomposites. ${ }^{71}$ In particular, PMMA has been used as a compatibiliser for various fillers in epoxy resins, such as glass fibre ${ }^{72}$ and nanoclays, ${ }^{73}$ in addition, PMMA is a well-known ATRP target. FLG-Br was therefore used as a precursor to obtain PMMA-grafted graphene as both a model system and for practical purposes. Using FLG-Br as the initiator molecule and following standard ATRP procedures in the literature, FLG-PMMA was obtained from the polymerisation of $\mathrm{MMA}^{74}$ (Scheme 1). Briefly, under inert conditions, distilled MMA was stirred with $\mathrm{CuBr}, \mathrm{CuBr}_{2}, \mathrm{PMDETA}$, and FLG$\mathrm{Br}$ in acetone, at $50{ }^{\circ} \mathrm{C}$ for $2 \mathrm{~h}$. FLG-PMMA was obtained as a black powder after washing with THF and drying under vacuum.

TGA-MS analysis of the product shows an increased mass loss (16.2 wt\%), and correspondingly larger grafting ratio (19.5 wt\%), as compared to FLG-Br, in the range 200 to $550{ }^{\circ} \mathrm{C}$ (Fig. 3a); the accompanying mass fragments correspond to PMMA polymer, including $\mathrm{m} / \mathrm{z} 41\left(-\mathrm{CH}_{2} \mathrm{C}\left(\mathrm{CH}_{3}\right)^{-}{ }^{+}\right), 59\left(-\mathrm{COOCH}_{3}{ }^{+}\right), 69\left(-\mathrm{CH}_{2}-\right.$ $\left.\mathrm{C}\left(\mathrm{CH}_{3}\right)(\mathrm{CO})-^{+}\right)$, and $100\left(-\mathrm{CH}_{2} \mathrm{C}\left(\mathrm{CH}_{3}\right)\left(\mathrm{COOCH}_{3}\right){ }^{-}\right)$. This weight loss temperature is also consistent with the expected thermal degradation of PMMA. ${ }^{8}$ XPS data for FLG-PMMA show an increase in oxygen content (Fig. S6 $\dagger$ ) with a composition of 92.6 at\% C, 6.9 at $\% \mathrm{O}$ and 0.5 at $\% \mathrm{Br}$ (Table 1); assuming, therefore, that approximately half the bromine addends served as initiators in the ATRP reaction, the polymer chains are estimated to have an average molecular weight in the range 370 to $590 \mathrm{Da}$, based on the $\mathrm{C} / \mathrm{Br}$ limits obtained from TGA and XPS (see ESI for 
a)

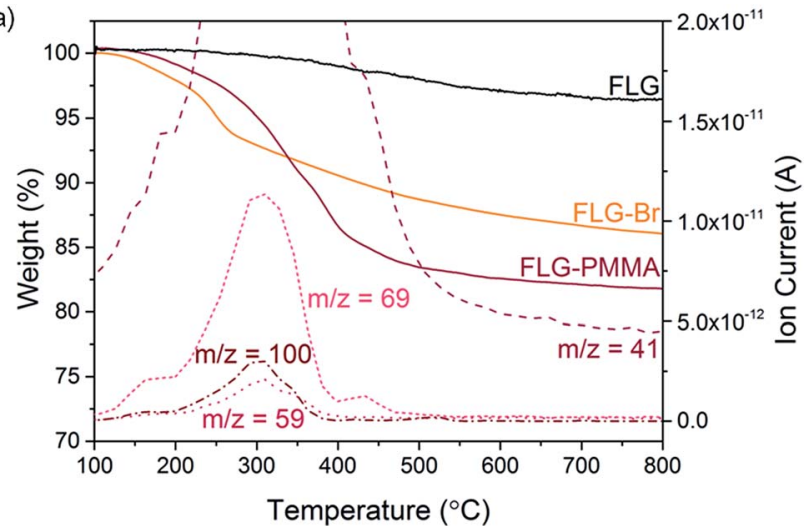

b)

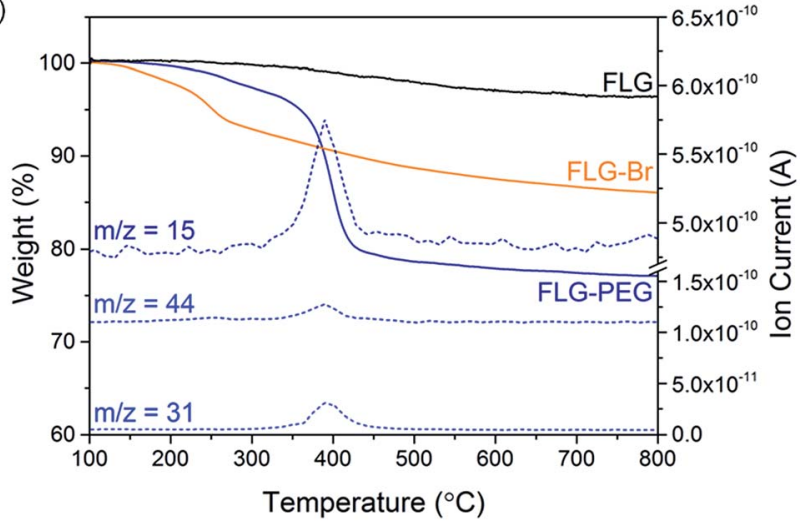

Fig. 3 (a) TGA-MS profile of FLG-PMMA, $m / z 41\left(-\mathrm{CH}_{2} \mathrm{C}\left(\mathrm{CH}_{3}\right)^{-}{ }^{+}\right), 59$ $\left(-\mathrm{COOCH}_{3}{ }^{+}\right), \quad 69 \quad\left(-\mathrm{CH}_{2} \mathrm{C}\left(\mathrm{CH}_{3}\right)(\mathrm{CO}){ }^{+}\right), \quad 100 \quad\left(-\mathrm{CH}_{2} \mathrm{C}\left(\mathrm{CH}_{3}\right)-\right.$ $\left.\left(\mathrm{COOCH}_{3}\right){ }^{+}\right)$; (b) TGA-MS profile of FLG-PEG, m/z $15\left(-\mathrm{CH}_{3}{ }^{+}\right), 31$ $\left(-\mathrm{OCH}_{3}{ }^{+}\right), 44\left(-\mathrm{CH}_{2} \mathrm{CH}_{2} \mathrm{O}-{ }^{+}\right)$.

calculations $\dagger$ ). The carbon and oxygen contents from XPS are in good agreement with calculated compositional values based on $\mathrm{C} /$ PMMA $=250, M_{\mathrm{n}} \sim 590$; the estimated grafting ratio from XPS is $21.4 \mathrm{wt} \%$, close to the value obtained by TGA. TGA-MS shows only a trace amount of THF in FLG-PMMA; the compositional values from XPS also suggest that no THF remains. Introduction of polymer on the graphene surface likely preserves an open pathway between layers, allowing evaporation of solvent.

Raman analysis of FLG-PMMA product shows that the initial density of defects in FLG-Br is hardly changed $\left(I_{\mathrm{D}} / I_{\mathrm{G}} 0.58 \pm 0.02\right.$ to $0.53 \pm 0.03$ ) after PMMA growth from the surface (Fig. 2 orange and red); therefore, no additional $\mathrm{sp}^{3}$ centres were introduced during this part of the reaction sequence. A reference experiment (FLG + MMA control) using FLG as the ATRP initiator, rather than FLG-Br, was carried out under the same conditions; the TGA-MS and Raman data (Fig. S7†) show that the reaction does not take place on the original FLG surface, demonstrating that brominated sites are necessary for polymerisation initiation.

An alternative route to polymer-grafted graphene, and further proof of the versatility of bromine functionalisation, is provided by nucleophilic substitution of the bromine addends with polyethylene glycol (Scheme 1). To obtain the PEGsubstituted product, FLG-Br was dispersed in dry THF and dichloromethane by brief and mild bath sonication. After addition of mPEG $\left(M_{\mathrm{W}} 2000 \mathrm{Da}\right)$, the suspension was stirred at
$40{ }^{\circ} \mathrm{C}$ for $2.5 \mathrm{~h}$, and FLG-PEG was obtained after filtering and washing with THF, water and ethanol, and drying under vacuum. The weight loss during TGA pyrolysis of FLG-PEG in the range $300-500{ }^{\circ} \mathrm{C}$ is accompanied by mass fragments which can be attributed to mPEG $\left(\mathrm{m} / \mathrm{z} 15\left(-\mathrm{CH}_{3}{ }^{+}\right), 31\left(-\mathrm{OCH}_{3}{ }^{+}\right), 44\right.$ $\left(-\mathrm{CH}_{2} \mathrm{CH}_{2} \mathrm{O}^{+}\right)$), confirming successful introduction of PEG on the graphene surface (Fig. 3b). The mass loss of $18.7 \mathrm{wt} \%$ implies an incomplete reaction of $\sim 1$ in 6 bromine addends (based on $\mathrm{C} / \mathrm{Br}$ from XPS); this partial substitution by MPEG may be explained by the steric bulk of the polymer chain which may prevent substitution of less accessible bromine-grafted sites. A large increase in oxygen content can be seen by XPS (Fig. S6 $\dagger$ ); $\mathrm{C}$ and $\mathrm{O}$ atomic percentages obtained by XPS (Table 1 ) are consistent with the proposed substitution of 1 in 6 addends, although the Br content is lower than expected, possibly due to the layer of long PEG chains on the surface. The grafting ratio from TGA of $23.8 \mathrm{wt} \%$ is in good agreement with that implied by the XPS data (26.2 wt\%). To explore whether substitution efficiency was affected by nucleophile size, FLG-Br was reacted with water under the same conditions for PEG substitution, to obtain FLG-OH. XPS data show that a greater degree of substitution occurs in this case, with over half (0.6) of the bromine addends replaced with hydroxyl groups (Table 1 ). The reduced TGA weight loss (6.1 wt\%, Fig. S8 $\dagger$ ) is also consistent with the substitution of this proportion of addends (see ESI for calculations $\dagger)$. Hydroxyl groups and water $(m / z 17,18)$ are detected by TGA-MS (Fig. S8 $\dagger$ ), as well as some residual solvent. However, bromine fragments are not seen, possibly because the low concentration cannot be detected by the MS; even in FLG-Br, the bromine $\mathrm{m} / \mathrm{z}$ fragments give only a very weak signal.

As is the case for FLG-PMMA, in the Raman spectra for the PEG- and OH-substituted products, the $I_{\mathrm{D}} / I_{\mathrm{G}}$ ratios of $0.56 \pm 0.03$ and $0.57 \pm 0.09$ (Fig. 2 blue, and Fig. S9†), respectively, indicate that no further defects are introduced during subsequent reaction, and that substitution occurs exclusively at existing grafted sites. Overall, the TGA, Raman and XPS data indicate that successful growth of PMMA by ATRP, or nucleophilic substitution, illustrated by mPEG and water, can be achieved from brominated FLG, without the creation of further defects.

\section{Exfoliation and dispersion}

Analysis of the $2 \mathrm{D}$ band in Raman spectra provides valuable information about the exfoliated nature of the different functionalised materials. The shape, intensity $\left(I_{2 \mathrm{D}}\right)$ and full width at half maximum $\left(\Gamma_{2 \mathrm{D}}\right)$ of the $2 \mathrm{D}$ peak can be used to evaluate the number of graphene layers present. ${ }^{61}$ FLG shows a symmetric $2 \mathrm{D}$ band at $2694 \mathrm{~cm}^{-1}$ with a $\Gamma_{2 \mathrm{D}}$ of $58 \mathrm{~cm}^{-1}$, characteristic of the loosely-stacked few-layer nature of the starting material. After bromine grafting, the $I_{2 \mathrm{D}} / I_{\mathrm{G}}$ ratio increases only slightly (from $0.97 \pm 0.04$ to $1.02 \pm 0.05$ ), since bromine addends are not large and do not significantly aid further exfoliation (Fig. 4a). Following PMMA functionalisation, this value increases again to $1.06 \pm 0.05$ suggesting that as ATRP progresses, graphene layers are pushed apart and prevented from restacking and aggregation by grafted polymer chains. An accompanying decrease in $\Gamma_{2 \mathrm{D}}$ (Fig. $4 \mathrm{~b}$ and Table S1 $\dagger$ ) also 

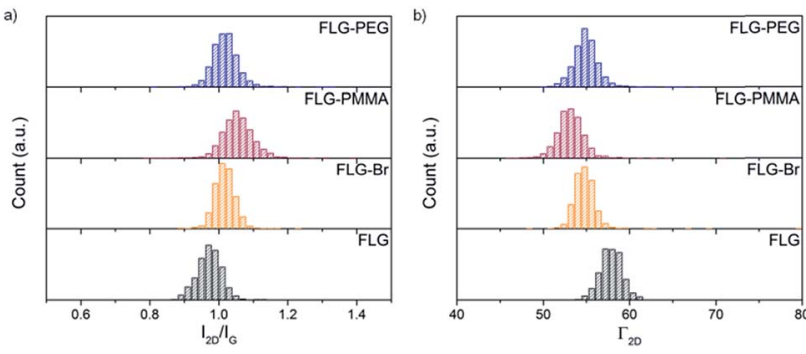

Fig. 4 Raman histograms of (a) $/ 2 \mathrm{D} / /_{\mathrm{G}}$ ratio, and (b) $\Gamma_{2 \mathrm{D}}$, of FLG (black) FLG-Br (orange), FLG-PMMA (red) and FLG-PEG (blue); $\lambda_{\text {exc }}=532 \mathrm{~nm}$.

implies that the grafted samples are more exfoliated than the starting material. ${ }^{75}$

In FLG-PEG, there is no significant change in either $I_{2 \mathrm{D}} / I_{\mathrm{G}}$ or $\Gamma_{2 \mathrm{D}}$ (Fig. 4) relative to the brominated FLG; it is likely that in this 'graft-to' reaction the large polymer chains cannot diffuse within the interlayer galleries so easily and therefore only substitute bromines grafted on the edges and surfaces of a fewlayer stack. As a result, no further exfoliation occurs, and the $I_{2 \mathrm{D}} / I_{\mathrm{G}}$ ratio remains unchanged. There is little change in $I_{2 \mathrm{D}} / I_{\mathrm{G}}$ after -OH substitution; hydroxyl addends are small and do not significantly prevent layer restacking after drying.

The dispersibility of the polymer-grafted graphenes was measured by UV-Vis absorbance (Fig. S10†). The samples were dispersed in different solvents by bath ultrasonication for 15 minutes and allowed to sediment over two days. The extinction coefficient $^{6}$ for dispersed graphene in solution $\left(\alpha_{660}=2460 \mathrm{~L}\right.$ $\mathrm{g}^{-1} \mathrm{~m}^{-1}$ ) was used to estimate the concentrations of the different solutions. Covalent grafting significantly improved the dispersibility of the material, with FLG-PMMA reaching a concentration of $200 \mu \mathrm{g} \mathrm{mL} \mathrm{mL}^{-1}$ in acetone, and FLG-PEG $255 \mu \mathrm{g}$ $\mathrm{mL}^{-1}$ in ethanol. In the case of FLG-PMMA, even the addition of low molecular weight polymer chains was enough to increase dispersibility six-fold in acetone. FLG-PEG was three and a half times more soluble than FLG in ethanol; despite a low grafting density, the longer mPEG chains could effectively stabilise the graphene sheets. Hydroxyl groups increased the solubility of FLG in water, from $8.51 \mu \mathrm{g} \mathrm{mL} \mathrm{m}^{-1}$ to $96.4 \mu \mathrm{g} \mathrm{mL} \mathrm{L}^{-1}$ (Table S2†).

\section{Conclusions}

Brominated graphene was synthesised by reacting exfoliated Na-based GICs with bromine, as confirmed by TGA-MS, XPS and Raman spectroscopy. This reductive method results only in a low concentration of $\mathrm{sp}^{3}$ defects and the underlying connectivity of the carbon network, and therefore graphene's intrinsic properties, ${ }^{60}$ are retained. The brominated FLG serves as a convenient and versatile precursor to modified graphenes. The FLG-Br can act as an initiator in the polymerisation of MMA by ATRP, resulting in PMMA-grafted graphene. In contrast to previous examples of ATRP on GO, CNTs and BNNTs, ${ }^{8,48-52}$ polymerisation is activated directly from the brominated graphene surface, rather than mediated by a specific grafted molecular group, providing an interesting new approach for ATRP reactions on nanocarbon surfaces. FLG-PMMA showed improved dispersibility in acetone, with the addition of only low molecular weight polymers; optimising polymerisation conditions could further increase dispersibility in various solvents. In contrast to direct anionic polymerisation from reduced graphite/graphenides, this methodology offers greater versatility with a wide choice of monomers including those that are unstable to graphenide, for example glycidyl methacrylate or aminoethyl methacrylate. In addition, the bromine addends may easily undergo nucleophilic substitutions, as illustrated by reactions with MPEG and water, without introducing any additional $\mathrm{sp}^{3}$ centres on the carbon framework. In this generalised reaction, brominated graphenes represent versatile, air-stable starting materials for the generation of grafted graphenes with a wide variety of functional moieties.

\section{Conflicts of interest}

There are no conflicts to declare.

\section{Acknowledgements}

The authors are grateful to Ignacio Villar-Garcia for his help interpreting and processing XPS data, to Catharina Paukner (FGV Cambridge Nanosystems Limited) for providing the FLG starting material, and thank the EPSRC (EP/G007314/1) for financial support.

\section{References}

1 X. Huang, X. Qi, F. Boey and H. Zhang, Chem. Soc. Rev., 2012, 41, 666-686.

2 W. K. Chee, H. N. Lim, N. M. Huang and I. Harrison, $R S C$ Adv., 2015, 5, 68014-68051.

3 K. Arapov, E. Rubingh, R. Abbel, J. Laven, G. de With and H. Friedrich, Adv. Funct. Mater., 2016, 26, 586-593.

4 Z. Liu, J. T. Robinson, X. Sun and H. Dai, J. Am. Chem. Soc., 2008, 130, 10876-10877.

5 R. S. Edwards and K. S. Coleman, Nanoscale, 2013, 5, 38-51. 6 Y. Hernandez, V. Nicolosi, M. Lotya, F. M. Blighe, Z. Sun, S. De, I. T. McGovern, B. Holland, M. Byrne, Y. K. Gun'Ko, J. J. Boland, P. Niraj, G. Duesberg, S. Krishnamurthy, R. Goodhue, J. Hutchison, V. Scardaci, A. C. Ferrari and J. N. Coleman, Nat. Nanotechnol., 2008, 3, 563-568.

7 X. Cui, C. Zhang, R. Hao and Y. Hou, Nanoscale, 2011, 3, 2118-2126.

8 G. Goncalves, P. A. A. P. Marques, A. Barros-Timmons, I. Bdkin, M. K. Singh, N. Emami and J. Gracio, J. Mater. Chem., 2010, 20, 9927-9934.

9 V. Georgakilas, M. Otyepka, A. B. Bourlinos, V. Chandra, N. Kim, K. C. Kemp, P. Hobza, R. Zboril and K. S. Kim, Chem. Rev., 2012, 112, 6156-6214.

10 J. Liu, J. Tang and J. J. Gooding, J. Mater. Chem., 2012, 22, 12435-12452.

11 L. Rodriguez-Perez, M. a. A. Herranz and N. Martin, Chem. Commun., 2013, 49, 3721-3735.

12 S. Eigler and A. Hirsch, Angew. Chem., Int. Ed., 2014, 53, $7720-7738$. 
13 C. K. Chua and M. Pumera, Chem. Soc. Rev., 2013, 42, 32223233.

14 J. M. Englert, K. C. Knirsch, C. Dotzer, B. Butz, F. Hauke, E. Spiecker and A. Hirsch, Chem. Commun., 2012, 48, 50255027.

15 J. R. Lomeda, C. D. Doyle, D. V. Kosynkin, W.-F. Hwang and J. M. Tour, J. Am. Chem. Soc., 2008, 130, 16201-16206.

16 E. Bekyarova, M. E. Itkis, P. Ramesh, C. Berger, M. Sprinkle, W. A. de Heer and R. C. Haddon, J. Am. Chem. Soc., 2009, 131, 1336-1337.

17 R. Sharma, J. H. Baik, C. J. Perera and M. S. Strano, Nano Lett., 2010, 10, 398-405.

18 M. Quintana, K. Spyrou, M. Grzelczak, W. R. Browne, P. Rudolf and M. Prato, ACS Nano, 2010, 4, 3527-3533.

19 D. R. Dreyer, S. Park, C. W. Bielawski and R. S. Ruoff, Chem. Soc. Rev., 2010, 39, 228-240.

20 J. Wang, Z. Shi, Y. Ge, Y. Wang, J. Fan and J. Yin, Mater. Chem. Phys., 2012, 136, 43-50.

21 M. Castelain, G. Martinez, G. Ellis and H. J. Salavagione, Chem. Commun., 2013, 49, 8967-8969.

22 J. Zheng, H.-T. Liu, B. Wu, C.-A. Di, Y.-L. Guo, T. Wu, G. Yu, Y.-Q. Liu and D.-B. Zhu, Sci. Rep., 2012, 2, 662.

23 R. Stine, W.-K. Lee, K. E. Whitener, J. T. Robinson and P. E. Sheehan, Nano Lett., 2013, 13, 4311-4316.

24 K. S. Coleman, A. K. Chakraborty, S. R. Bailey, J. Sloan and M. Alexander, Chem. Mater., 2007, 19, 1076-1081.

25 P. C. Eklund, N. Kambe, G. Dresselhaus and M. S. Dresselhaus, Phys. Rev. B: Solid State, 1978, 18, 70697079.

26 L. G. Bulusheva, A. V. Okotrub, E. Flahaut, I. P. Asanov, P. N. Gevko, V. O. Koroteev, Y. V. Fedoseeva, A. Yaya and C. P. Ewels, Chem. Mater., 2012, 24, 2708-2715.

27 J. Gao, F. Bao, Q. Zhu, Z. Tan, T. Chen, H. Cai, C. Zhao, Q. Cheng, Y. Yang and R. Ma, Polym. Chem., 2013, 4, 16721679.

28 T. Sainsbury, M. Passarelli, M. Naftaly, S. Gnaniah, S. J. Spencer and A. J. Pollard, ACS Appl. Mater. Interfaces, 2016, 8, 4870-4877.

29 D. Hines, M. H. Rummeli, D. Adebimpe and D. L. Akins, Chem. Commun., 2014, 50, 11568-11571.

30 J. F. Friedrich, S. Wettmarshausen, S. Hanelt, R. Mach, R. Mix, E. B. Zeynalov and A. Meyer-Plath, Carbon, 2010, 48, 3884-3894.

31 O. Jankovsky, P. Simek, K. Klimova, D. Sedmidubsky, S. Matejkova, M. Pumera and Z. Sofer, Nanoscale, 2014, 6, 6065-6074.

32 F. Hof, F. Hauke and A. Hirsch, Chem. Commun., 2014, 50, 6582-6584.

33 D. Voiry, O. Roubeau and A. Penicaud, J. Mater. Chem., 2010, 20, 4385-4391.

34 A. Molina-Ontoria, M. N. Chaur, M. E. Plonska-Brzezinska and L. Echegoyen, Chem. Commun., 2013, 49, 2406-2408.

35 S. Chakraborty, J. Chattopadhyay, W. Guo and W. E. Billups, Angew. Chem., Int. Ed., 2007, 46, 4486-4488.

36 D. E. Nixon and G. S. Parry, J. Phys. D: Appl. Phys., 1968, 1, 291.
37 Y. Wang, P. Puech, I. Gerber and A. Pénicaud, J. Raman Spectrosc., 2014, 45, 219-223.

38 M. Zanini, S. Basu and J. E. Fischer, Carbon, 1978, 16, 211212.

39 T. Morishita, A. J. Clancy and M. S. P. Shaffer, J. Mater. Chem. A, 2014, 2, 15022-15028.

40 J. Chattopadhyay, A. Mukherjee, S. Chakraborty, J. Kang, P. J. Loos, K. F. Kelly, H. K. Schmidt and W. E. Billups, Carbon, 2009, 47, 2945-2949.

41 J. M. Englert, C. Dotzer, G. Yang, M. Schmid, C. Papp, J. M. Gottfried, H.-P. Steinrück, E. Spiecker, F. Hauke and A. Hirsch, Nat. Chem., 2011, 3, 279-286.

42 H. S. Leese, L. Govada, E. Saridakis, S. Khurshid, R. Menzel, T. Morishita, A. J. Clancy, E. R. White, N. E. Chayen and M. S. P. Shaffer, Chem. Sci., 2016, 7, 2916-2923.

43 R. A. Schäfer, J. M. Englert, P. Wehrfritz, W. Bauer, F. Hauke, T. Seyller and A. Hirsch, Angew. Chem., Int. Ed., 2013, 52, 754-757.

44 N. Rubio, H. Au, H. S. Leese, S. Hu, A. J. Clancy and M. S. P. Shaffer, Macromolecules, 2017, 50, 7070-7079.

45 Y.-S. Ye, Y.-N. Chen, J.-S. Wang, J. Rick, Y.-J. Huang, F.-C. Chang and B.-J. Hwang, Chem. Mater., 2012, 24, 2987-2997.

46 F. Liang, J. M. Beach, K. Kobashi, A. K. Sadana, Y. I. VegaCantu, J. M. Tour and W. E. Billups, Chem. Mater., 2006, 18, 4764-4767.

47 T. Sarbu, K.-Y. Lin, J. Ell, D. J. Siegwart, J. Spanswick and K. Matyjaszewski, Macromolecules, 2004, 37, 3120-3127.

48 H. Roghani-Mamaqani, RSC Adv., 2015, 5, 53357-53368.

49 M. Ejaz, S. C. Rai, K. Wang, K. Zhang, W. Zhou and S. M. Grayson, J. Mater. Chem. C, 2014, 2, 4073-4079.

50 S. H. Lee, D. R. Dreyer, J. An, A. Velamakanni, R. D. Piner, S. Park, Y. Zhu, S. O. Kim, C. W. Bielawski and R. S. Ruoff, Macromol. Rapid Commun., 2010, 31, 281-288.

51 H. Kong, C. Gao and D. Yan, J. Am. Chem. Soc., 2004, 126, 412-413.

52 D. Baskaran, J. W. Mays and M. S. Bratcher, Angew. Chem., Int. Ed., 2004, 43, 2138-2142.

53 E. Widenkvist, D. W. Boukhvalov, S. Rubino, S. Akhtar, J. Lu, R. A. Quinlan, M. I. Katsnelson, K. Leifer, H. Grennberg and U. Jansson, J. Phys. D: Appl. Phys., 2009, 42, 112003.

54 D. A. Young, Carbon, 1977, 15, 373-377.

55 G. A. Saunders, A. R. Ubbelohde and D. A. Young, Proc. $R$. Soc. London, Ser. A, 1963, 271, 499-511.

56 J. S. Culik and D. D. L. Chung, Mater. Sci. Eng., 1980, 44, 129137.

57 E. Papirer, R. Lacroix, J.-B. Donnet, G. Nanse and P. Fioux, Carbon, 1994, 32, 1341-1358.

58 J. Li, L. Vaisman, G. Marom and J.-K. Kim, Carbon, 2007, 45, 744-750.

59 A. E. Mansour, S. Dey, A. Amassian and M. H. Tanielian, ACS Appl. Mater. Interfaces, 2015, 7, 17692-17699.

60 A. J. Marsden, P. Brommer, J. J. Mudd, M. A. Dyson, R. Cook, M. Asensio, J. Avila, A. Levy, J. Sloan, D. Quigley, G. R. Bell and N. R. Wilson, Nano Res., 2015, 8, 2620-2635. 
61 A. C. Ferrari, J. C. Meyer, V. Scardaci, C. Casiraghi, M. Lazzeri, F. Mauri, S. Piscanec, D. Jiang, K. S. Novoselov, S. Roth and A. K. Geim, Phys. Rev. Lett., 2006, 97, 187401.

62 A. C. Ferrari and D. M. Basko, Nat. Nanotechnol., 2013, 8, 235-246.

63 K. C. Knirsch, J. M. Englert, C. Dotzer, F. Hauke and A. Hirsch, Chem. Commun., 2013, 49, 10811-10813.

64 S. Eigler, F. Hof, M. Enzelberger-Heim, S. Grimm, P. Müller and A. Hirsch, J. Phys. Chem. C, 2014, 118, 7698-7704.

65 J. M. Englert, P. Vecera, K. C. Knirsch, R. A. Schäfer, F. Hauke and A. Hirsch, ACS Nano, 2013, 7, 5472-5482.

66 A. C. Ferrari and J. Robertson, Phys. Rev. B: Condens. Matter Mater. Phys., 2000, 61, 14095-14107.

67 L. G. Cançado, A. Jorio, E. H. M. Ferreira, F. Stavale, C. A. Achete, R. B. Capaz, M. V. O. Moutinho, A. Lombardo, T. S. Kulmala and A. C. Ferrari, Nano Lett., 2011, 11, 3190-3196.
68 S. Deng, Y. Zhang, A. H. Brozena, M. L. Mayes, P. Banerjee, W.-A. Chiou, G. W. Rubloff, G. C. Schatz and Y. Wang, Nat. Commun., 2011, 2, 382.

69 A. Eckmann, A. Felten, A. Mishchenko, L. Britnell, R. Krupke, K. S. Novoselov and C. Casiraghi, Nano Lett., 2012, 12, 3925-3930.

70 T. Gatti, N. Vicentini, M. Mba and E. Menna, Eur. J. Org. Chem., 2016, 2016, 1071-1090.

71 R. J. Young, I. A. Kinloch, L. Gong and K. S. Novoselov, Compos. Sci. Technol., 2012, 72, 1459-1476.

72 D. Olmos, K. Bagdi, J. Mózcó, B. Pukánszky and J. GonzálezBenito, J. Colloid Interface Sci., 2011, 360, 289-299.

73 M. Hernandez, B. Sixou, J. Duchet and H. Sautereau, Polymer, 2007, 48, 4075-4086.

74 T. Sarbu, K.-Y. Lin, J. Spanswick, R. R. Gil, D. J. Siegwart and K. Matyjaszewski, Macromolecules, 2004, 37, 9694-9700.

75 L. M. Malard, M. A. Pimenta, G. Dresselhaus and M. S. Dresselhaus, Phys. Rep., 2009, 473, 51-87. 